\title{
Prevalence of suicidal ideation and attempted suicide in the general population of Ethiopia: a systematic review and meta-analysis
}

\author{
Berhanu Boru Bifftu ${ }^{*^{*}}$ D, Bewket Tadesse Tiruneh ${ }^{1}$, Berihun Assefa Dachew ${ }^{3,4}$ and Yonas Deressa Guracho ${ }^{2}$
}

\begin{abstract}
Background: In Ethiopia, in spite of the high burden of suicide related-adverse effect, substantial variability in the reported prevalence of individual studies about suicidal ideation and attempted suicide; there is no national level epidemiological evidence. Thus, the present study aimed to determine the pooled prevalence of suicide ideation and suicidal attempt in the general population.

Methods: We followed the PRISMA Guidelines to report the results of the finding. Databases including: PubMed/ Medline, SCOPUS, CINAHL (EBSCOhost), African Journal Online (AJOL) and African Indexed Medicus (AIM) were searched. Heterogeneity was assessed by Cochrane chi-square $\left(X^{2}\right)$ and quantified by $I^{2}$ statistics test. Sensitivity test and subgroup analysis performed. Publication bias was tested by funnel plots and Egger's test. Effect size was calculated by random effects model.

Results: A total of 12 studies for suicidal ideation and 10 studies for attempted suicide were included in the study. The prevalence of suicidal ideation and attempted suicide were ranged from 1 to $55 \%$ and $0.6 \%$ to $14 \%$ respectively. The 12-month pooled prevalence of suicidal ideation and suicidal attempt were $9 \%(5-16 \%), I^{2}=99.64 \%, p<0.001$ and $4 \%(1-8 \%), I^{2}=98.11 \%, p<0.001$ respectively. The lifetime pooled prevalence of attempted suicide was found to be $4 \%(3-6 \%)$. We found evidence of significant heterogeneity for suicidal ideation $\left[l^{2}=99.64 \%, p<0.001\right]$ and attempted suicide $\left[I^{2}=98.11 \%, p<0.001\right]$. The subgroup analysis could not identified source of heterogeneity. The sensitivity analysis showed that none of the point estimates was outside of the overall $95 \% \mathrm{Cl}$ for suicidal ideation and attempted suicide. No evidence of publication bias from the visual inspection of the funnel plot for suicidal ideation and [Egger's test $(P=0.174)$ ] and attempted suicide [Egger's test $(P=0.318)]$.
\end{abstract}

Conclusion: High prevalence of suicidal ideation and attempted suicide were observed in the general population of Ethiopia. These suggest the need of strengthening the awareness of suicidal behaviours and evaluate the effectiveness of the national health strategy in addressing the issues of suicidal behaviours.

Keywords: Ethiopia, Suicide, Attempted suicide, Suicidal ideation

*Correspondence: berhanuboru@gmail.com

${ }^{1}$ School of Nursing, University of Gondar College of Medicine and Health Science, Gondar, Ethiopia

Full list of author information is available at the end of the article

\section{Background}

Suicide is serious public health problem [1-3] that has multiple, complex and inter-related psychological, social and economical impacts across the population [4]. Globally, one million people died by suicide per year (one

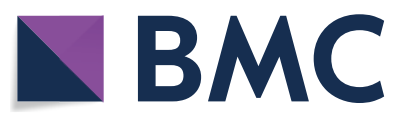

(c) The Author(s) 2021. This article is licensed under a Creative Commons Attribution 4.0 International License, which permits use, sharing, adaptation, distribution and reproduction in any medium or format, as long as you give appropriate credit to the original author(s) and the source, provide a link to the Creative Commons licence, and indicate if changes were made. The images or other third party material in this article are included in the article's Creative Commons licence, unless indicated otherwise in a credit line to the material. If material is not included in the article's Creative Commons licence and your intended use is not permitted by statutory regulation or exceeds the permitted use, you will need to obtain permission directly from the copyright holder. To view a copy of this licence, visit http://creativeco mmons.org/licenses/by/4.0/. The Creative Commons Public Domain Dedication waiver (http://creativecommons.org/publicdomain/ zero/1.0/) applies to the data made available in this article, unless otherwise stated in a credit line to the data. 
person every $40 \mathrm{~s}$ ) [2, 5-8]. It contributes to $1.4 \%$ of the global burden of disease and the second cause of death for individuals age between 15 and 29 years $[9,10]$. The World Health Organization (WHO) Mental Health Action Plan 2013 to 2020 aims to reduce on the full range of suicidal phenomena including: suicidal ideation and suicidal behaviour by $10 \%$ [11]. Suicidal ideation and attempts suicide were the primary risk for ended individual's life $[1,2,8,12]$. Evidence showed that 50 to $75 \%$ of suicidal ideation and attempts suicide were reported to primary health care prior to took his/her life [13-15]. The global lifetime prevalence of suicidal ideation in the general population was ranged from 3.1 to $56 \%$ [10]. The corresponding figures in Africa and Ethiopia were 18.6-20.7 and $6 \%$ to $55 \%[4,12-18,18-23]$ respectively. Similarly, the prevalence of attempted suicide was ranged from 0.4 to $5.1 \%$ globally $[10,24,25], 18.6-20.7$ in Africa and $1.4 \%$ to $19 \%$ in Ethiopia [4, 12-22].

By its nature, suicide is a complex phenomenon that involves the interactions of factors at different level such as: system level (access to means of suicide and health care), individual level (demographic features: female gender, younger age, lower education level, unmarried status) and community level (stigma and discrimination) $[4,26]$. For example, systematic reviews and meta-analysis showed factors associated with suicidal behaviours include: psychological, psychiatric, biological and stressful life events $[4,27,28]$. Recently published systematic reviews and meta-analysis among the general population in Europe showed that female gender, age over 65 years, unemployment, low social support, adulthood adversity, childhood adversity, family history of mental disorder, affective disorder, major depression, anxiety disorders and substance use were associated with attempted suicide $[27,28]$. In addition to these, the lack of standardized methods for data collection, the legal status of suicide and its impact on reporting, lack of resources, stigma, cultural criticism $[2,7,8,23,29-31]$ and the association of suicide with a criminal act and insurance policies have been affected the true figure of suicidal behaviours in the general population and compared its extent to an iceberg, where only the tip is visible; while the majority hidden under the surface $[4,10]$.

In Ethiopia despite several inconsistent individual studies with ranged lifetime prevalence of suicidal ideation (1\% to $55 \%)$, attempted suicide $(0.6 \%$ to $14 \%)$ [17, $32-39]$, death by suicide (7.2 to 8.4 per 100,000$)$ [40] and lack of accessible health care facilities, skilled man power, stigma, taboo and cultural criticism toward people with suicidal behaviours; priorities are given for other cause of death like infectious related death and the issue of national level epidemiological evidence of death by suicide has little attention. The contextual, epidemiological understanding of suicidal ideation and attempted suicide is an important first step to design culture specific suicide interventions. Therefore, the present systematic review and meta-analysis aimed to determine the pooled prevalence of suicidal ideation and attempted suicide in the general population of Ethiopia.

\section{Methods \\ Study protocol registration and publication}

This systematic review and meta-analysis was conducted according to a priori registered (CRD42018112836) and published protocol [41] with slight modification to the original designed published proposal. This modification is (i) related to the populations (i. e clinical, general population, high school and university students) and (ii) suicide-related outcomes (as suicide morbidity and suicide mortality). Because of the distinct characteristics of clinical patients and students from the general populations and suicide mortality as one end of suicidal behaviours from suicide morbidity as the other end, in the present study, we presented a separate manuscript for the general population and suicide morbidity (suicidal ideation and attempted suicide).

The findings in this report presented within, the first domain of suicide prevention activity [universal activities or interventions] that targeted the general public that has not been identified on the basis of individual risk [42, 43]. According to a framework of Gordon's continuum of care model, prevention, treatment and maintenance were the three model of care $[44,45]$. These are (i) universal activities or interventions, the focus of this study that targeted the general public has not been identified on the basis of individual risk, (ii) selected or targeted interventions that aim to reduce the risk among specific high-risk groups and (iii) indicated interventions include the treatments of targeted individuals presenting with suicidal behaviour. This model was previously adopted to guide identification of population groups and individuals with differing prevention needs, and alignment of these needs with appropriate policies and programmes for suicide prevention [45]. Results were reported in accordance with the Preferred Reporting Items for Systematic Review and Meta-Analysis (PRISMA) [46].

\section{Search strategy}

A compressive search of electronic databases: PubMed/ Medline, SCOPUS, CINAHL (EBSCOhost), African Journal Online (AJOL) and African Indexed Medicus (AIM) were searched using keywords, Medical Subject Headings (MeSH) terms. Search strings were established using "AND" and/"OR" Boolean operators for each databases. Moreover, to avoid missing of relevant studies like preprints and grey literatures, web of science (Google 
and Google Scholars) searched. Finally, reference lists of included articles were searched. All searches were performed until November, 20, 2020. Sample search query for PubMed/MEDLINE: ((suicide [MeSH Terms]) OR (suicide) OR ("suicidal behavior" [MeSH Terms]) OR ("suicidal behavior") OR ("suicide attempt" [MeSH Terms]) OR ("suicide attempt") OR ("suicide thoughts" [MeSH Terms]) OR ("suicide thoughts") OR ("suicide ideation" [MeSH Terms]) OR ("suicide ideation")) AND (("associated factors" [MeSH Terms]) OR ("associated factors") OR "risk factor" [MeSH Terms]) OR ("risk factors") OR (risk [MeSH Terms]) OR (risk) OR ("determinant factors" [MeSH Terms]) OR ("determinants factors")) AND ((epidemiology [MeSH Terms]) OR (epidemiology) OR (prevalence [MeSH Terms]) OR (prevalence) OR (incidence [MeSH Terms]) OR (incidence)) AND (("general population" [MeSH Terms]) OR ("general population") OR (community [MeSH Terms]) OR (community)) AND Ethiopia. Similarly, Scopus, and CINAHL (EBSCO host) databases were searched using similar search terms tailored to each database. There is no language and publication year restriction applied.

\section{Selection of studies}

All studies retrieved through search strategy were imported in to EndNote X7 (Thomson Reuters, New York, USA) and removed duplicated studies. The study selection process had three stages: the first stage was screened based on the title and abstract of retrieved references against the outcome. The second stage was based on the full-text to determine its applicability to the study aim/data's clarity. Finally, studies were screened based on the eligibility criteria. Two reviewers (YDG and BBB) independently screened the studies. Disagreements were solved by discussion.

\section{Definition of concepts}

Suicidal ideation refers to thoughts, fantasies and wishes about ending of one's own life [1, 29, 47]. Overlapping terms in the concept of suicide attempt: (i) attempted suicide/self-harm/suicidal intent, refer to a deliberated direct destruction of body tissues with a conscious suicidal intent, (ii) non-suicidal attempt/selfinjury, refer to a deliberated direct destruction of body tissues without a conscious suicidal intent $[4,11]$ and (iii) para-suicide, is a term that does not refer to the intention and covers behaviors that can vary from suicidal gestures to serious attempted suicide but unsuccessful to kill oneself [48]. Most standard guidelines and evidence focus on self-harm irrespective of the intent. In line with other similar studies, in this study, suicidal attempt was defined irrespective of its intent and assessment tools $[4,11,48]$. In the current study, for the assessment of suicidal ideation and attempted suicide, (i) majority ( $75 \%$ of ideation and $90 \%$ of attempted) of the included studies used Composite International Diagnostic Interview (CIDI). In Ethiopia, CIDI had established reliability and acceptability [26]. In CIDI, a hierarchy of single item questions used for the assessment of suicidal behaviours: For example, suicidal ideation was assessed by the statement: "Have you thought of taking your life in the past 12 months?"; suicide plan (asked of those who had suicidal ideation): "Did you ever make a plan for taking your own life at any time in the past 12 months?"; attempted suicide (asked to those who answered they had ideation or a plan): "Have you attempted to take your own life in the past 12 months?", (ii) The remaining $25 \%$ of suicidal ideation was assessed by Self-Report Questionnaire-20 $(\mathrm{n}=3)$. SRQ-20 had established reliability and acceptability test in Ethiopia as a screening tool to identify probable common mental disorders. The scale contains an item on suicidal thoughts. Similarly, the remaining $10 \%$ of attempted suicide used Suicidal Behaviour Questionnaire-Revised ( $\mathrm{n}=1)$.

\section{Eligibility criteria}

According to the PICOS acronym, the following inclusion criteria were used: Participants $(\mathrm{P})$ : all studies carried out among the general population, Intervention (I): not applicable, Comparison (C): not applicable, Outcomes $(\mathrm{O})$ : prevalence of suicidal ideation and suicidal attempt, Study design (S): observational studies (crosssectional and cohort/longitudinal) were included and Study setting: all studies carried out in Ethiopia were included in the study. Exclusion criteria were: (1) case reports, conference, reviews and abstracts were excluded (2). Studies conducted among clinical population (e.g. psychiatric, physical problems including HIV/AIDs), students and specific population group such as: one sex, prisoners, refugees and street (homeless), (3) For those studies reporting the overall results of suicidal behavior and not reported separately for suicidal ideation and suicidal attempt and (4) studies not conducted in Ethiopia were excluded.

\section{Data extraction}

Data were extracted from the eligible studies using a pre-conceived and piloted data extraction Microsoft Excel format. Data were simultaneously extracted by two independent reviewers (BBB and YDG). The included data items from each article include: name of the first author, year of publication, study area, study design, data 
collection tool, sample size and number of cases/prevalence including for each sex.

\section{Quality assessment}

The quality of each article was evaluated using the Joanna Briggs Institute (JBI) quality appraisal criteria adapted for reporting the prevalence data for cross-sectional studies [49]. This JBI quality assessment tool has nine items with response option of "yes", "no", "unclear" and "not applicable". These items include: sample frame, sampled participants, sample size, description of study subjects and setting in details, data analysis with sufficient coverage of the identified sample, use of valid method in the identification of conditions, measurements of outcome with a standard, reliable way, appropriateness of statistical analysis and the adequate of response rate. The overall quality of each study was determined by the summed score of all items to generate a study specific global score. Studies were included in analysis if the sum of the overall individual study score is $\geq 50 \%$ of the quality assessment checklist criteria's. Finally, based on the overall mean score (8) we categorized individual study as high quality $(\geq 8)$ and low quality $(<8)$. Two reviewers (YDG and $\mathrm{BBB}$ ) independently screened the studies. Disagreements were solved by discussion.

\section{Data synthesis and statistical analysis}

The extracted data into Microsoft Excel Database were exported into Stata 14 that we installed packages for meta-analyses online. Metaprop command in Stata employed to calculate the pool point prevalence estimate with $95 \%$ CIs by a random-effects model [50] using the Dersimonian and Laird transformed inverse variance method [51]. Test for Heterogeneity was performed using Cochrane chi-square $\left(\chi^{2}\right)$ and the $I^{2}$ statistics. $I^{2}$ value greater than $50 \%$ was considered as indicative of substantial heterogeneity [52]. Two separate meta-analyses were performed for the overall outcome (suicidal ideation and suicidal attempt). To assess the possible source of heterogeneity, sensitivity analyses [53] and subgroup analyses by sex, sample size, study setting, publication years and time frame were conducted. Publication bias was tested by the visual inspection of funnel plot [54] and egger test [55]. A p-value $<0.1$ was considered as indicative of statistically significant publication bias. The findings of this study summarized and presented using texts, forest plots, tables and summery of descriptive statistics.

\section{Results}

The initial database search resulted in 2759 publications. Additional, 23 studies were located from the other sources. This resulted in 2782 records. Of these records,
1329 were excluded because of duplication. Of the remaining 1453, 824 studies were excluded by their title and abstract reading which were not related to the outcome and 579 articles were not full text. Finally, 37 publications were excluded based on the eligibility criteria and objective (Fig 1).

\section{Study characteristics}

In this study, a total of 13 studies were included in the systematic review and meta-analysis. Of these, 12 studies reported suicidal ideation and 10 studies attempted suicide. These studies were carried out in five different regions of the country: South Nation and Nationality People (SNNP) $(n=5)$, Addis Ababa $(n=2)$, Oromia $(\mathrm{n}=2)$ and Harar $(\mathrm{n}=1)$ and Tigray $(\mathrm{n}=1)$. All studies used cross-sectional study design. The sample sizes of included studies ranged from 351 to 10,203 for suicidal ideation and 351 to 10,468 for attempted suicide. Studies were published between 1999 and 2020. For the assessment of suicidal ideation, the vast majority (75\%) used the Composite International Diagnostic Interview (CIDI) and the remaining three studies used Self-Report Questionnaire 20 (SRQ-20). Similarly, 90\% of attempted suicide was assessed by CIDI and the remaining one study used Suicidal Behaviour Questionnaire-Revised (SBQ-R) (Table 1).

\section{Quality of included studies}

The quality of included articles in this systematic review and meta-analysis is shown in Table 2. Eight-five percent of the studies used an appropriate sample frame. All studies $(100 \%)$ used a standard instrument, described the study subjects, setting and employed appropriate statistical analysis. The list scored quality item was observed in the sample size (31\%). The overall quality score of included studies had a mean score of 7.6 (ranged 7-9). Ten articles (77\%) were high-quality and three studies were low quality.

\section{Prevalence of suicidal ideation and attempted suicide Suicidal ideation}

The prevalence of suicidal ideation was ranged from 1 to $55 \%$ (Table 1). The 12-month pooled prevalence estimate of suicidal ideation was $9 \%(5 \%-16 \%),\left[\mathrm{I}^{2}=99.64 \%\right.$, $\mathrm{p}<0.001, \mathrm{n}=12$ ], (Fig. 2). No lifetime reported prevalence of suicidal ideation.We found evidence of significant heterogeneity $\left[\mathrm{I}^{2}=99.64 \%, \mathrm{p}<0.001\right.$, (Fig. 2)]. The subgroup analysis by study setting, sample size, publication year and quality of study could not identified source of heterogeneity; however, the highest pooled prevalence was considered among studies that carried out between 1999 to 2010 [19\% (95\% CI 5-39\%)] (Table 3). The sensitivity analysis showed that none of the point estimates 


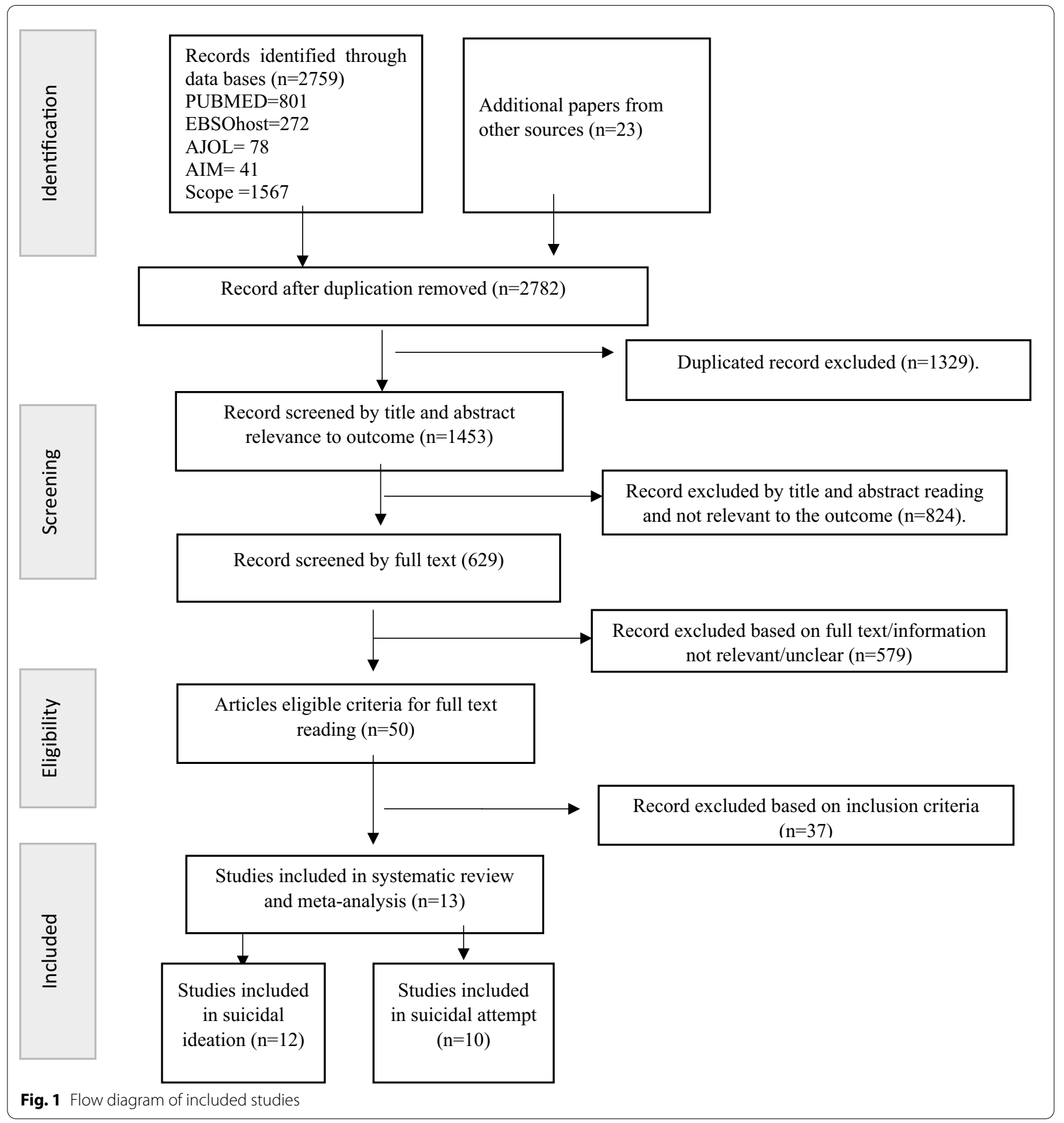

was outside of the overall $95 \% \mathrm{CI}$. No evidence of publication bias from the visual inspection of the funnel plot (Fig. 3) and [Egger's test $(\mathrm{P}=0.174)$ ].

\section{Attempted suicide}

In this systematic review and meta-analysis, the prevalence of attempted suicide was ranged from $0 . \%$ to $14 \%$. The 12-month and lifetime pooled prevalence of attempted suicide was $4 \%(1-8 \%),\left[\mathrm{I}^{2}=98.11, \mathrm{p}<0.00\right.$, $\mathrm{n}=10]$ (Fig. 4) and 4\% (1-7\%), $\left[\mathrm{I}^{2}=90.07, \mathrm{p}<0.001, \mathrm{n}=3\right]$ (Fig. 5) respectively. We found a statistical significant evidence of heterogeneity across studies $\left[\mathrm{I}^{2}=98.11 \%\right.$, $\mathrm{p}<0.001$, (Fig. 4) for 12-month] and $\left[\mathrm{I}^{2}=99.07, \mathrm{p}<0.001\right.$, (Fig. 5) for lifetime]. The subgroup analysis by study setting, sample size, publication year and quality of study 
Table 1 Characteristics of included studies

\begin{tabular}{|c|c|c|c|c|c|c|c|c|}
\hline \multirow[t]{2}{*}{ Author } & \multirow[t]{2}{*}{ Year } & \multirow[t]{2}{*}{ Setting } & \multirow[t]{2}{*}{ population } & \multirow[t]{2}{*}{ tools } & \multirow[t]{2}{*}{ Time frame } & \multirow[t]{2}{*}{ Total } & \multicolumn{2}{|c|}{ Case (percent) } \\
\hline & & & & & & & Ideation & Attempt \\
\hline Jordan & 2017 & SNNP & Community & CIDI & 12-month & 1486 & $104(7)$ & $55(3.7)$ \\
\hline Fekadu & 2016 & SNNP & Community & CIDI & 12-month & 1484 & $93(6)$ & $56(4)$ \\
\hline Fekadu & 2014 & SNNP & Community & $\mathrm{CIDI}$ & 12-month & 1497 & $202(13)$ & $21(1.4)$ \\
\hline Fekadu & 2007 & SNNP & Community & $\mathrm{CIDI}$ & 12-month & 1504 & $829(55)$ & $221(14.7)$ \\
\hline Fekadu & 2008 & Oromia & Community & CIDI & 12-month & 351 & $125(36)$ & $18(5)$ \\
\hline Alem A & 1999 & SNNP & Community & $\mathrm{CIDI}$ & Lifetime & 10,468 & $314(3)$ & $332(3.2)$ \\
\hline Kebede D & 1999 & Addis Ababa & Community & CIDI & Lifetime & 10,203 & $277(3)$ & $275(2.7)$ \\
\hline Nyundo & 2019 & Oromia & Community & CIDI & 12-month & 1059 & $64(6)$ & $38(4)$ \\
\hline Nyundo & 2019 & Harar & Community & $C|D|$ & 12-month & 951 & $11(1)$ & $6(0.6)$ \\
\hline Mekonen & 2020 & Addis Ababa & Community & SBQ-R & Lifetime & 481 & - & $47(10)$ \\
\hline Gelay & 2012 & Addis Ababa & Community & SRQ-20 & 12-month & 2180 & $47(2)$ & - \\
\hline Hunduma & 2017 & Harar & Community & SRQ-20 & 12-month & 901 & $93(10.3)$ & - \\
\hline Abbay & 2018 & Tigray & Community & SRQ-20 & 12-month & 260 & $12(4.6)$ & - \\
\hline
\end{tabular}

CIDI Composite International Diagnostic Interview, SBQ- $R$ Suicidal Behaviour Questionnaire-Revised and, SRQ-20 Self-Report Questionnaire 20, SNNP South Nation and Nationality People

Table 2 JBI Critical Appraisal Checklist for the included studies in the analysis

\begin{tabular}{|c|c|c|c|c|c|c|c|c|c|c|c|}
\hline \multirow[t]{2}{*}{ Authors } & \multirow[t]{2}{*}{ Year } & \multicolumn{9}{|c|}{ Quality domain } & \multirow[t]{2}{*}{ Overall Score } \\
\hline & & Q1 & Q2 & Q3 & Q4 & Q5 & Q6 & Q7 & Q8 & Q9 & \\
\hline Jordan & 2017 & Y & Y & Y & Y & Y & Y & Y & Y & N & 8 \\
\hline Fekadu & 2016 & Y & Y & Y & Y & Y & Y & Y & Y & Y & 9 \\
\hline Fekadu & 2014 & Y & Y & Y & Y & Y & Y & Y & Y & Y & 9 \\
\hline Fekadu & 2007 & Y & Y & Y & Y & Y & Y & Y & Y & Y & 8 \\
\hline Fekadu & 2008 & Y & Y & Y & Y & Y & Y & Y & Y & Y & 8 \\
\hline Alem A & 1999 & Y & Y & Y & Y & Y & N & Y & Y & Y & 8 \\
\hline Kebede D & 1999 & Y & Y & Y & Y & Y & N & Y & Y & Y & 8 \\
\hline Nyundo & 2019 & Y & Y & Y & Y & Y & $\mathrm{N}$ & Y & Y & N & 7 \\
\hline Nyundo & 2019 & Y & Y & Y & Y & Y & N & Y & Y & N & 7 \\
\hline Mekonen & 2020 & Y & Y & N & Y & Y & Y & Y & Y & Y & 8 \\
\hline Gelay & 2012 & Y & Y & $N$ & Y & $N$ & Y & Y & Y & Y & 7 \\
\hline Hunduma & 2017 & $N$ & Y & Y & Y & Y & $Y$ & Y & Y & Y & 8 \\
\hline Abbay & 2018 & $N$ & Y & Y & Y & Y & Y & Y & Y & $Y$ & 8 \\
\hline
\end{tabular}

A. Q1-Q9 represents questions used to assess the quality of included studies, which are listed below

Q1.Was the sample frame appropriate to address the target populations?

Q2. Were the study participants sampled in appropriate way?

Q3. Was the sample size adequate?

Q4. Were the study subjects and setting described in details?

Q5. Was the data analysis conducted with sufficient coverage of the identified sample?

Q6. Was a valid method used in the identification of conditions?

Q7. Was the condition measured in a standard, reliable way for all participants?

Q8. Was there an appropriate statistical analysis?

Q9. Was the response rate adequate, and if not, was the low response rate managed appropriately?

B. Y, yes; N, no; U, unclear; NA, not applicable 


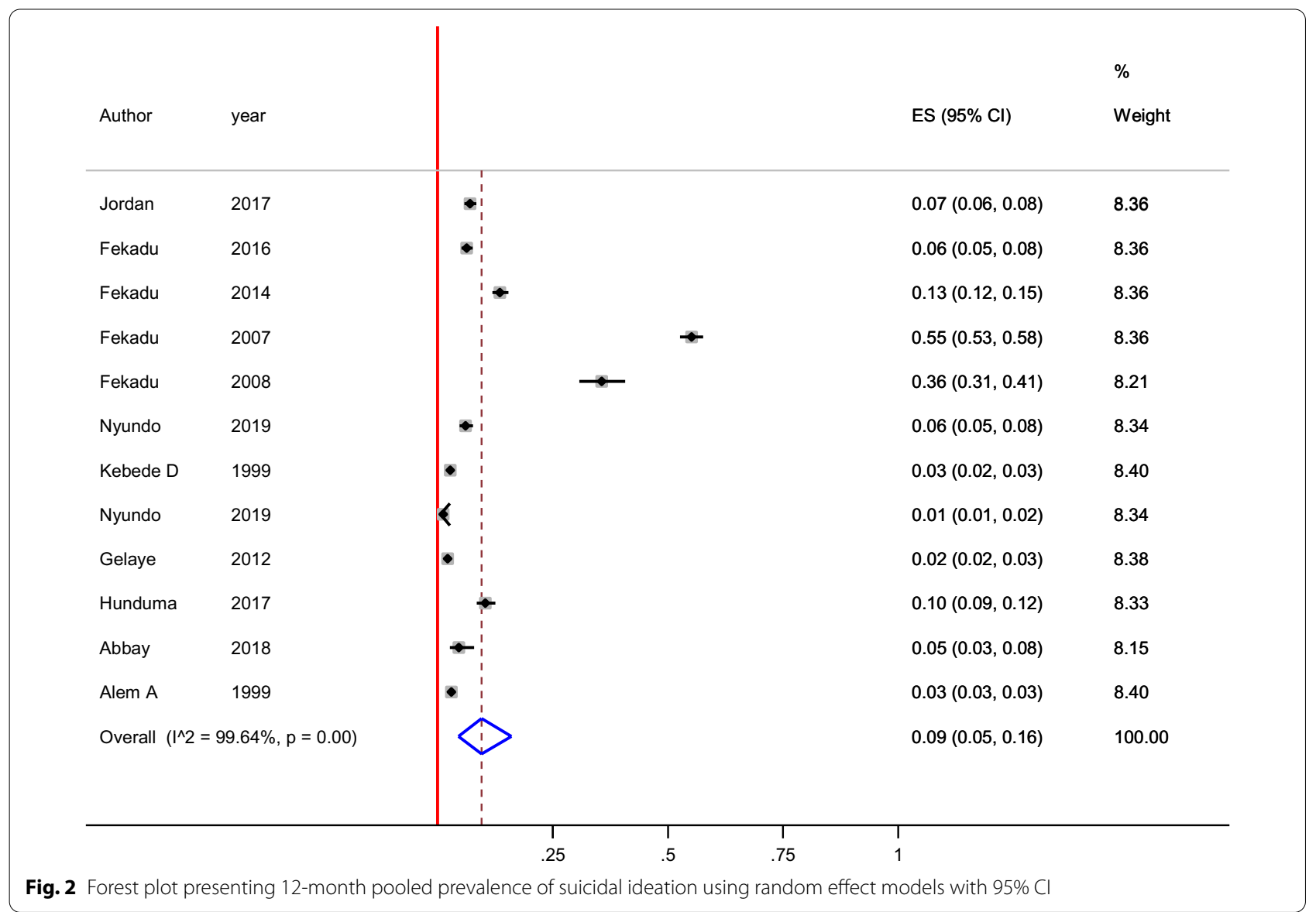

could not identified source of heterogeneity; however, the highest pooled prevalence was observed among studies used CIDI assessment tool [14\% (95\% CI 4-30\%)] (Table 3). The sensitivity analysis showed that none of the point estimates was outside of the overall $95 \%$ CI. No evidence of publication bias from the visual inspection of the funnel plot (Fig. 6) and [Egger's test $(P=0.745)]$.

\section{Sex distribution of suicidal ideation and attempted suicide}

Regarding the sex distribution, a total of seven studies (one suicidal ideation and six attempted suicide) reported data on the sex distribution of suicidal behaviours. From this, the sex distribution of suicidal ideation was $2.7 \%$ for female and 2.9 for male. On the other hand, the pooled effect of six studies for attempted suicide showed that females were around 1.19 times (AOR $=95 \% \mathrm{CI}$ $\left.0.41-1.96, \mathrm{I}^{2}=0 \%, \mathrm{p}=0.987\right)$ more likely experienced attempted suicide as compared to males; yet, insignificant (Fig. 7).

\section{Discussion}

To the best of the authors' knowledge, there is no previous systematic review and meta-analysis has been reported about the pooled prevalence of suicidal ideation and attempted suicide in Ethiopia. The observed prevalence of suicidal ideation [ $1 \%$ to $55 \%]$ ) and attempted suicide $[0.6 \%$ to $14 \%]$ ) in this study is within the reported prevalence of suicidal ideation and attempted suicide across the world [10]. Comparing with other similar studies, the pooled prevalence of suicidal ideation [9\% (5-16\%), for 12-month] and attempted suicide [(4\% (1-8\%, for $12-$ month) and $(4 \%(1-7 \%)$, for lifetime] in this study is similar with the 12-month pooled prevalence of suicidal ideation in developing countries (8.1\%) and the lifetime pooled prevalence of attempted suicide in Europe [2.88\% $(2.15 \%-3.6)$ and other meta-analysis of community study [6\% (4-9\%)] [4]. On the other hand, the observed12-month and lifetime pooled prevalence attempted suicide in this study is higher than the pooled prevalence meta-analysis in Europe $[(0.57$ (0.1-0.6) for 12-month] and [2.88\% (2.15-3.6\%) for lifetime] [27, 28], China $[0.2 \%(0.1-0.6 \%)$ for 12 -month] and [0.8\% (0.7$0.9 \%)$ for lifetime] [56], and lifetime pooled prevalence 
Table 3 Subgroup analysis of suicidal ideation and suicidal attempt by study population, sample size, setting, publication year, tool and time frame

\begin{tabular}{|c|c|c|c|c|c|c|c|c|c|c|}
\hline \multirow[t]{2}{*}{ Subgroup } & \multicolumn{5}{|c|}{ Suicidal ideation } & \multicolumn{5}{|c|}{ Suicidal attempt } \\
\hline & No. of studies & $\begin{array}{l}\text { Pooled } \\
\text { prevalence }\end{array}$ & $95 \% \mathrm{Cl}$ & $P^{2}$ & $p$-value & No. of studies & $\begin{array}{l}\text { Pooled } \\
\text { prevalence }\end{array}$ & $95 \% \mathrm{Cl}$ & $P^{2}$ & p-value \\
\hline \multicolumn{11}{|l|}{ Sample size } \\
\hline Small (<median) & 5 & 9 & $2-19$ & $98.99 \%$ & $<0.001$ & 4 & 5 & $1-11$ & $91.16 \%$ & $<0.001$ \\
\hline Large ( $\geq$ median) & 7 & 10 & $3-19$ & $99.80 \%$ & $<0.001$ & 6 & 3 & $2-5$ & $98.47 \%$ & $<0.001$ \\
\hline \multicolumn{11}{|l|}{ Study setting } \\
\hline SNNP & 5 & 14 & $2-32$ & $99.78 \%$ & $<0.001$ & 5 & 5 & $2-8$ & $98.74 \%$ & $<0.001$ \\
\hline Oromia & 2 & 11 & $10-13$ & - & - & 2 & 4 & $3-5$ & - & - \\
\hline Addis Ababa & 2 & 3 & $2-3$ & - & - & 2 & 3 & $2-3$ & - & - \\
\hline Harar & 1 & 5 & $4-6$ & - & - & 1 & 2 & $1-3$ & - & - \\
\hline Tigray & 1 & 5 & $3-8$ & - & - & - & - & - & - & - \\
\hline \multicolumn{11}{|l|}{ Publication year } \\
\hline 1999-2010 & 4 & 19 & $5-39$ & $99.89 \%$ & $<0.001$ & 5 & 5 & $2-8$ & $99.86 \%$ & $<0.001$ \\
\hline 2011-2020 & 8 & 6 & $3-9$ & $97.86 \%$ & $<0.001$ & 5 & 4 & $2-6$ & $97.58 \%$ & $<0.001$ \\
\hline \multicolumn{11}{|l|}{ Quality of studies } \\
\hline High & 9 & 14 & $6-26$ & $99.79 \%$ & $<0.001$ & 8 & 5 & $3-7$ & $96.14 \%$ & $<0.001$ \\
\hline Low & 3 & 4 & $2-8$ & $96.76 \%$ & $<0.001$ & 2 & 2 & $1-3$ & - & - \\
\hline \multicolumn{11}{|l|}{ Tool } \\
\hline$C|D|$ & 7 & 14 & $4-30$ & $99.68 \%$ & $<0.001$ & 7 & 4 & $1-8$ & $97.86 \%$ & $<0.001$ \\
\hline SRQ-20 & 5 & 4 & $3-6$ & $95.54 \%$ & $<0.001$ & - & - & - & - & - \\
\hline SRB-R & - & - & - & - & - & 1 & 3 & $2-4$ & - & - \\
\hline \multicolumn{11}{|l|}{ Timeframe } \\
\hline 12-month & 12 & & & & & 7 & 4 & $1-8$ & & \\
\hline Lifetime & - & - & - & - & - & 3 & 4 & $3-6$ & $97.86 \%$ & $<0.001$ \\
\hline
\end{tabular}

$95 \% \mathrm{Cl}$ represents the $95 \%$ Confidence Interval for prevalence and $\mathrm{I}^{2}$ true heterogeneity

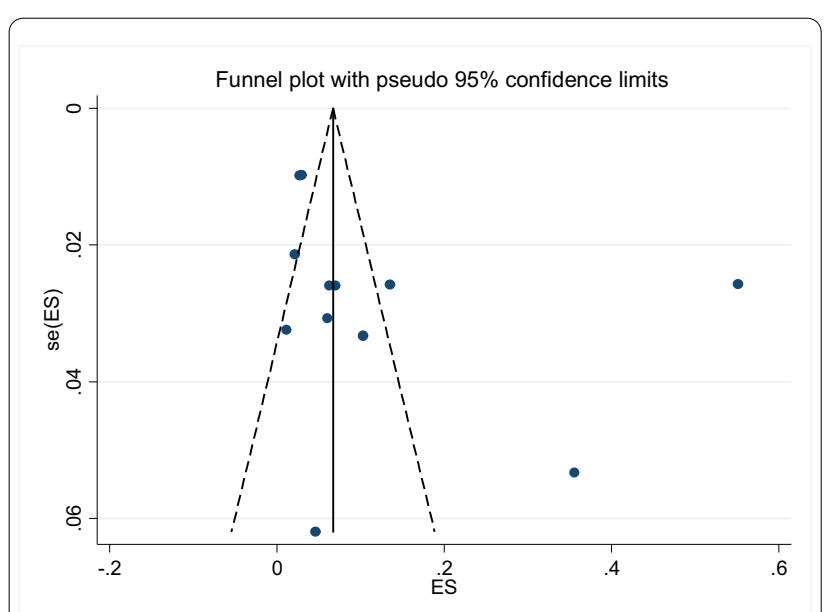

Fig. 3 Funnel plot with pseudo $95 \% \mathrm{Cl}$ presented the publication bias for suicidal ideation

across 17 countries [2.7\%] [26]. The possible explanation for the variation may be due to the difference in educational level, accessibility of information, socio-economic status, and availability of resource, community's attitude toward suicidal behavior and geographical areas. In Ethiopian, for family with natural death strong social support system has been practiced; yet, fear associated with the stigma, cultural taboo, cultural criticism affect community's attitude, practice and help seeking behaviours.

The observed high prevalence of attempted suicide in female; yet, insignificant is consistent with study carried out in the general populations of China [57]. This may be attributed to the high prevalence of sexual violence and depression in female as compared to males or they might have the history of abortion which is associated with higher rates of suicide. This is supported by other evidences [58-60]. In Ethiopia, evidence showed the association of sexual violence, early marriage (before 18 years) with suicidal attempt [60]. Another possible explanation may be due to the variation in gender specific cause of suicidal behaviours. For example, a recently published systematic review and meta-analysis showed that eating disorder, posttraumatic stress disorder, bipolar disorder, violence, depressive symptoms and previous history of abortion as female specific risks for suicidal attempts [58, 


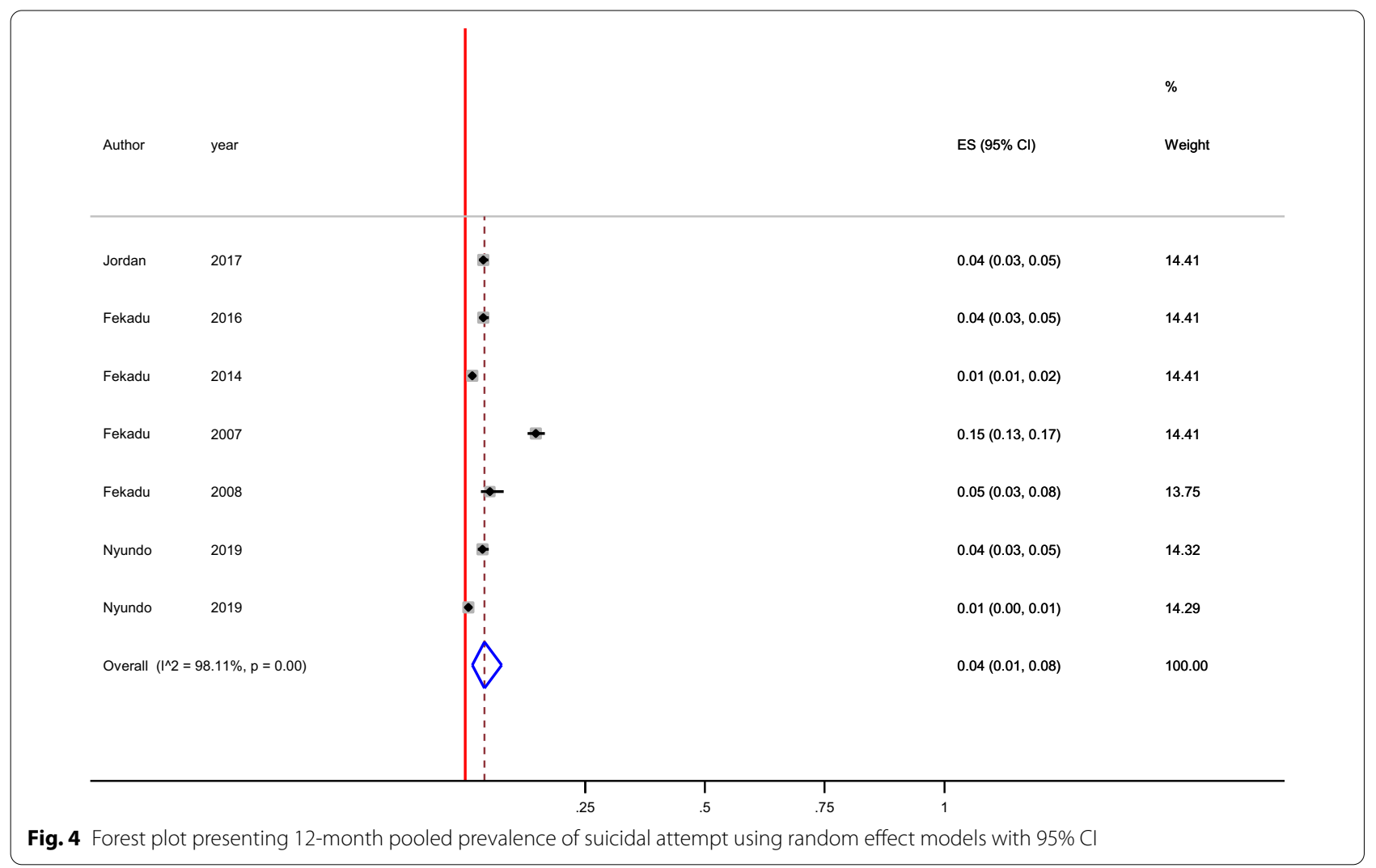

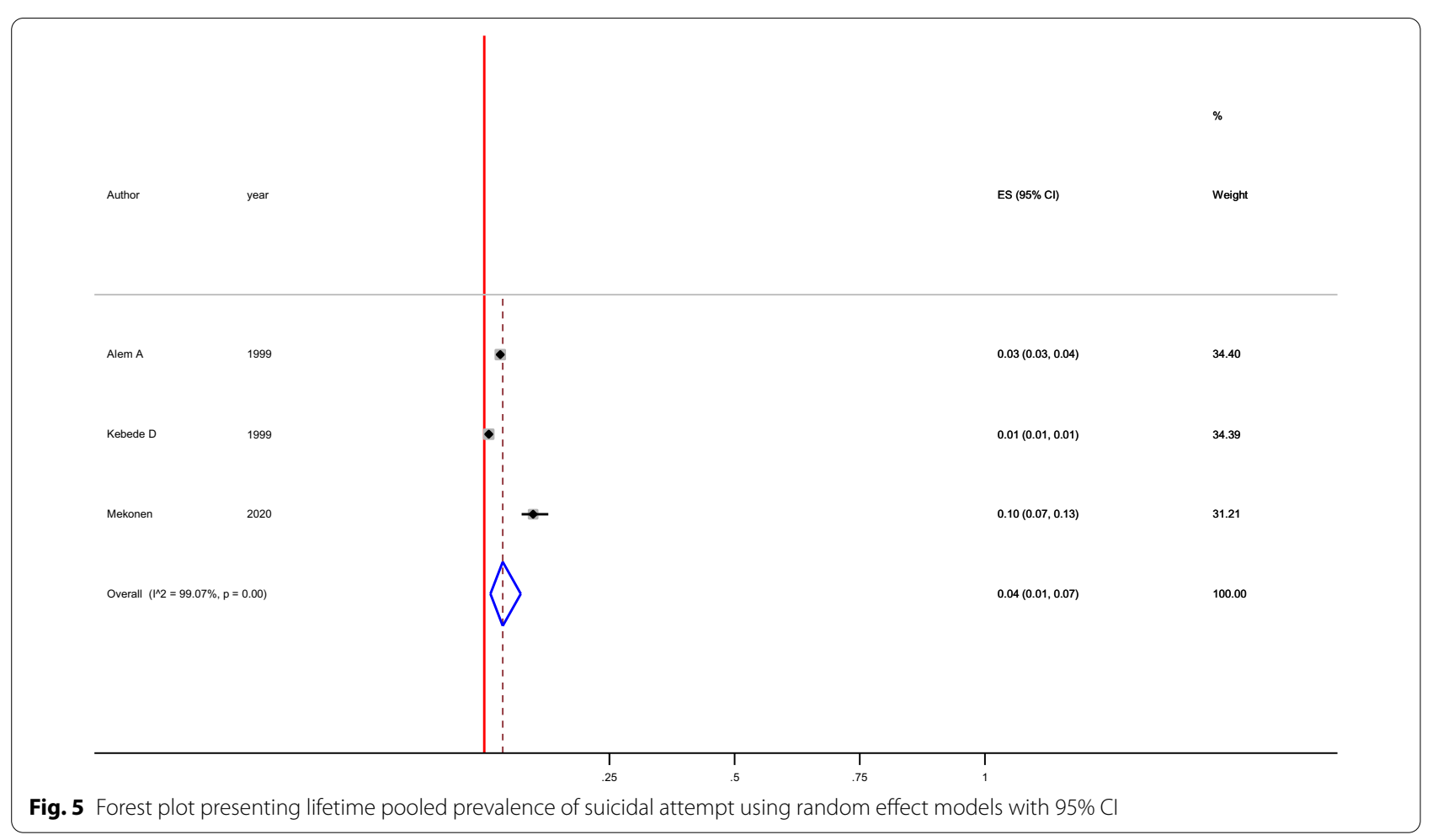




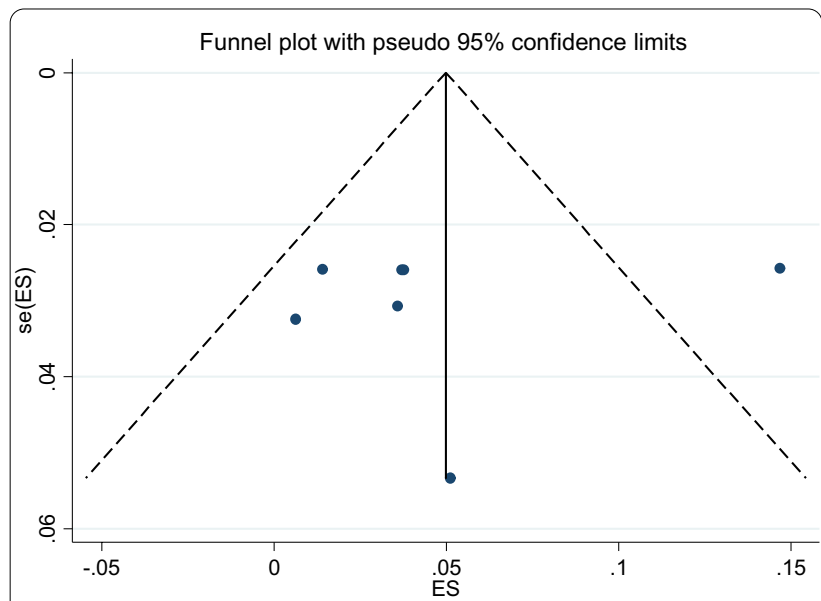

Fig. 6 Funnel plot with pseudo 95\% Cl presented the publication bias for suicidal attempt

59]; where, majority of these factors are the major problems in Ethiopia.

Therefore, the finding of this study implies; the existence of high burden of suicidal ideation and suicidal attempt in the general population. This suggests (i) the need of community based screening particularly for those who have difficult to access healthcare facilities (ii) the needs of strengthening community awareness toward suicidal behaviours. Suicide is a tragedy that can be prevented only when one can stand for each other. (iii) at the moment, however, the HMIS does not have a priority indicators for intentional harm, the WHO comprehensive action plan for mental health is suggested to report suicide rate as an urgent priority and (v) suggest the needs of evaluating the effectiveness of the national mental health strategy in addressing suicidal behaviours. This is supported by studies, which showed factors such as absence of psychotic, have unmanageable debts, selfemployed, higher levels of social problem solving were associated with the difficult to prevent suicide [13-15, 25].

\section{Strengths and limitations of the study}

This is the first systematic review and meta-analysis about suicidal ideation and attempted suicide in Ethiopia, and use of reference lists and Google Scholar to include all the available studies. However, limitations like exclusion of clinical patient, students, prisoner and the observed heterogeneity in spite of the subgroup analysis may limit the representativeness of the study.

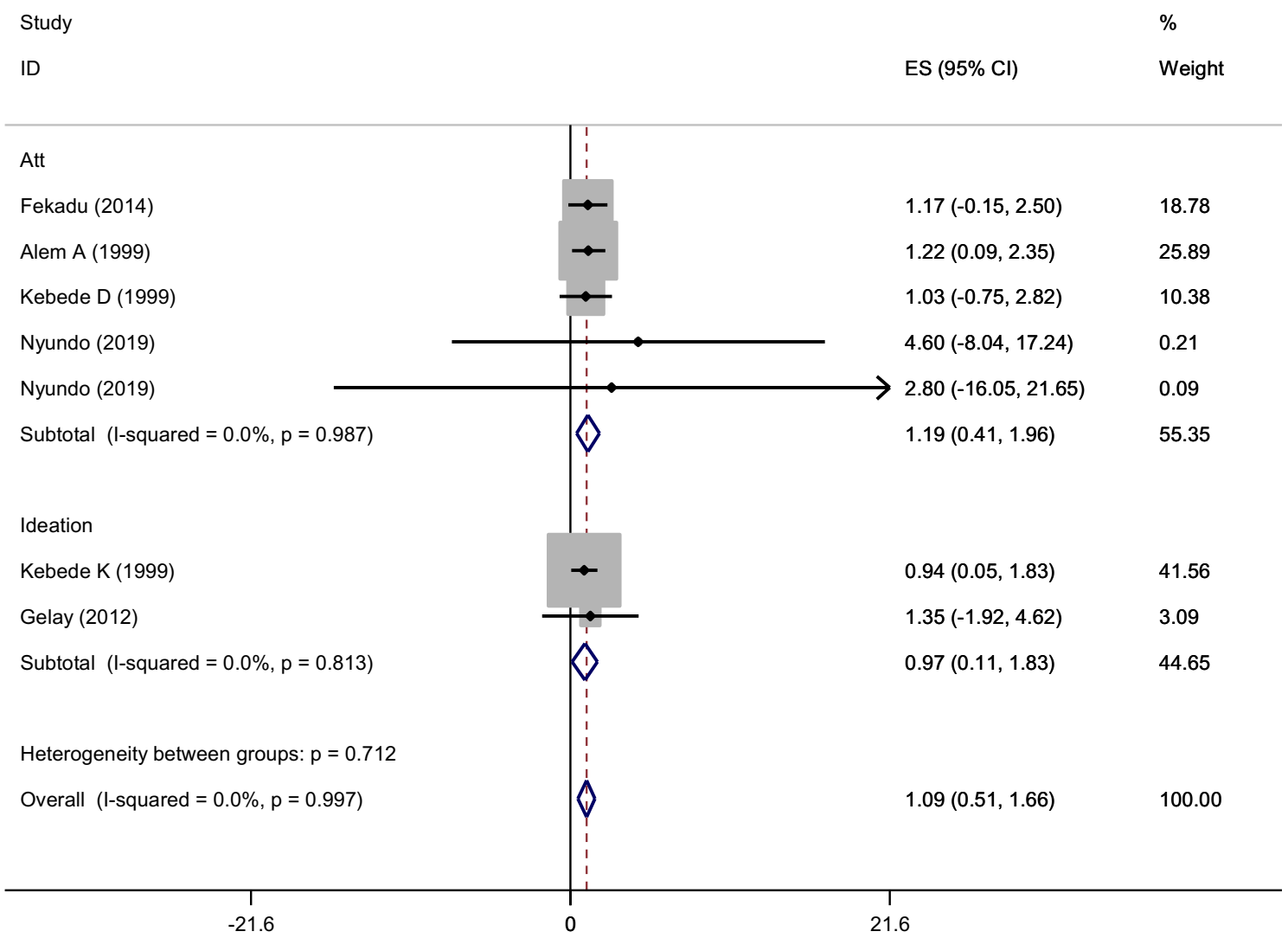

Fig. 7 Forest plot presenting the adjusted odds ratios with $95 \% \mathrm{Cls}$ on the association of females sex with suicidal attempt 


\section{Conclusion}

High prevalence of suicidal ideation and attempted suicide were observed in the general population of Ethiopia. This suggests the need of strengthening the awareness of suicidal behaviours. It also suggests the needs of evaluating the effectiveness of the national health strategy in addressing suicidal behaviours among non-clinical population.

\begin{abstract}
Abbreviations
$\mathrm{Cl}$ : Confidence interval; CIDI: Composite international diagnostic interview; CRD: Centre for reviews and dissemination; SRQ-20: Self-Report Questionnaire 20; DSH: Deliberate self-harm; DSM-IV: Diagnostic and Statistical Manual of Mental Disorders 4th edition; EJHD: Ethiopian Journal of Health Development; EJHS: Ethiopian Journal of Health Science; EMBASE: ExcerptaMedica database; $\mathrm{I}^{2}$ : Index of heterogeneity; ICD-10: Tenth Revision of the International Statistical Classification of Diseases and Related Health Problems; MEDLINE: Medical Literature Analysis and Retrieval System Online; MeSH: Medical Subject Headings; NSSI: Non-suicidal self-injury; OR: Odd ratio; PRISMA: Preferred Reporting Items for Systematic Review and Meta-Analysis; PROSPERO: International Prospective Register of Systematic Reviews; SBQ-R: Suicidal Behaviour Questionnaire-Revised; SNNP: South Nation and Nationality People; WHO: World Health Organization.
\end{abstract}

\section{Acknowledgements}

Authors' gratitude goes to University of Gondar for office arrangement and motivational support to conduct this systematic review and meta-analysis.

\section{Authors' contributions}

$B B B$ designed the systematic review and meta-analysis in collaboration with BAD, BTT, LDG and YDG. BBB developed the search strategy and drafted the protocol. BAD, BTT, LDG and YDG improved the drafted systematic review and meta-analysis. BBB, YDG, LDG, and BAD provided their expertise to the section of suicidal behaviours and methodological section. BBB and YDG performed search strategy and conducted data selection and extraction. All authors were involved in data analysis and interpretation of the results and write up the manuscript. All authors read and approved the final manuscript.

\section{Funding}

This research received no grant from any funding agency in the public, commercial or not-for-profit sectors.

\section{Availability of data}

Not applicable.

\section{Declarations}

Ethics approval and consent to participants

Not applicable.

\section{Consent for publication}

Not applicable.

\section{Competing interests}

None declared.

\footnotetext{
Author details

${ }^{1}$ School of Nursing, University of Gondar College of Medicine and Health Science, Gondar, Ethiopia. ${ }^{2}$ Department of Psychiatry, College of Medicine and Health Science, Bahir Dar University, Bahir Dar, Ethiopia. ${ }^{3}$ Department of Epidemiology and Biostatistics, Institute of Public Health, College of Medicine and Health Sciences, University of Gondar, Gondar, Ethiopia. ${ }^{4}$ Institute for Social Science Research, The University of Queensland, Brisbane, QLD. 4068, Australia.
}

Received: 18 September 2020 Accepted: 6 March 2021

Published online: 24 March 2021

\section{References}

1. Jans TVT, Taneli Y, Warnke A. Suicide and self-harming behaviour. Suicide and self-harming behaviour. In: Rey JM, editor. IACAPAP e-Textbook of child and adolescent mental health. Geneva: International Association for Child and Adolescent Psychiatry and Allied Professions; 2018.

2. Health WM. Prevention of suicidal behaviours: a task for all. http://www. who.int/mental_health/prevention/suicide/background. Accessed 15 Oct 2017.

3. Amare T. Prevalence and associated factors of suicide ideation and attempt among adolescent high school students in Dangila Town, Northwest Ethiopia. Int J Ment Health Syst. 2018;2018:7631453.

4. Dong M, Zeng LN, Lu L, Xiao-Hong L, Ungvari GS, Ng CH, Chow IH, Zhang $L$, Zhou Y, Yu-Tao X. Prevalence of suicide attempt in individuals with major depressive disorder: a meta-analysis of observational surveys. Psychol Med. 2018. https://doi.org/10.1017/S0033291718002301.

5. Stone DM, Holland KM, Bartholow B, Crosby AE, Davis S, Wilkins N. Preventing suicide: a technical package of policies, programs, and practices. Atlanta: National Center for Injury Prevention Control, Centers for Disease Control and Prevention; 2017. p. 1-62.

6. American. Foundation for suicide prevention. https://afsp.org/about-suici de/suicidestatistics/ Accessed 15 Oct 2017.

7. WHO. Global Health Observatory Data Repository. http://apps.who.int/ gho/data/nodemainMHSUICIDE?lang=en. Accessed 14 Oct 2017.

8. Suicide W. Fact Sheet. http://www.who.int/mediacentre/factsheets/ fs398/en/. Accessed 10 Oct 2017.

9. Zubrick SR, Hafekost J, Johnson SE, Lawrence D, Saw S, Sawyer M, et al. Suicidal behaviours: prevalence estimates from the second Australian child and adolescent survey of mental health and wellbeing. Aust N Z J Psychiatry. 2016;50(9):899-910.

10. Nock MK, Borges G, Bromet EJ, Cha CB, Kessler RC, Lee S. Suicide and suicidal behavior. Epidemiol Rev. 2008;30(1):133-54. https://doi.org/10. 1093/epirev/mxn002.

11. World Health Organization. Mental health action plan 2013-2020. Geneva, Switzerland: World Health Organization. 2013. https://apps.who. int/iris/bitstream/handle/10665/89966/9789241506021_eng.pdf.

12. WHO. Figure: male: female ratio of age-standardized suicide rates (per 100000 population). Geneva:WHO; 2015.

13. Law YW, Wong PW, Yip PS. Suicide with psychiatric diagnosis and without utilization of psychiatric service. BMC Public Health. 2010;10(431):1-11.

14. Barnes LSIR, Kresnow MJ. Help-seeking behavior prior to nearly lethal suicide attempts. Suicide Life Threat Behav. 2001;32(1):68-75.

15. Pagura JFS, Katz LY, Sareen J. Help seeking and perceived need for mental health care among individuals in Canada with suicidal behaviors. Psychiatr Serv. 2009;60(7):943-9.

16. Asfaw H, Yigzaw N, Yohannis Z, Fekadu G. Alemayehu Y Prevalence and associated factors of suicidal ideation and attempt among undergraduate medical students of Haramaya University, Ethiopia: a cross sectional study. PLoS ONE. 2020;15(8):e0236398. https://doi.org/10.1371/journal. pone.0236398.

17. Hunduma G, Girma M, Digaffe T, Weldegebreal F, Tola A. Prevalence and determinants of common mental illness among adult residents of Harari Regional State Eastern Ethiopia. Pan Afr Med J. 2017;28(262):1-16.

18. Jordans M, Rathod S, Fekadu A, Medhin G, Kigozi F, Kohrt B, Luitel N, Petersen I, Shidhaye R, Ssebunnya J, Patel V. Suicidal ideation and behaviour among community and health care seeking populations in five low- and middle-income countries: a cross-sectional study. Epidemiol Psychiatr Sci. 2018;27(4):393-402.

19. Misganaw A, Haregu TN, Deribe K, Tessema GA, Deribew A, Melaku YA, Amare AT, Abera SF, Gedefaw M, Dessalegn M, Lakew Y. National mortality burden due to communicable, non-communicable, and other diseases in Ethiopia, 1990-2015: findings from the Global Burden of Disease Study 2015. Popul Health Metrics. 2017;15:29. https://doi.org/10.1186/ s12963-017-0145-1. 
20. Fekadu A, Medhin G, Selamu M, Shiferaw T, Hailemariam M, Rathod SD, et al. Non-fatal suicidal behaviour in rural Ethiopia: a cross-sectional facility- and population-based study. BMC Psychiatry. 2016;16:75.

21. Alem A, Kebede D, Jacobsson L, Kullgren G. Suicide attempts among adults in Butajira Ethiopia. Acta Psychiatr Scand Suppl. 1999;397:70-6.

22. Kebede $D$, Alem A. Suicide attempts and ideation among adults in Addis Ababa Ethiopia. Acta Psychiatr Scand Suppl. 1999;397:35-9.

23. Mars SB, Hjelmeland H, Gunnell D. Suicidal behaviour across the African continent: a review of the literature. BMC Public Health. 2014;14(606):1-15.

24. Castellvía EA. Longitudinal association between self-injurious thoughts and behaviors and suicidal behavior in adolescents and young adults: a systematic review with meta-analysis. J Affect Disord. 2017;215:37-8.

25. Ribeiro JD, Franklin JC, Fox KR, Bentley KH, Kleiman EM, Chang BP. Nock MK Self-injurious thoughts and behaviors as risk factors for future suicide ideation, attempts, and death: a meta-analysis of longitudinal studies. Psychol Med. 2016;46(2):225-36.

26. Nock MKBG, Bromet EJ, et al. Cross-national prevalence and risk factors for suicidal ideation, plans and attempts. Br J Psychiatry. 2008;192:98-105.

27. Bernal MHJ, Bernert S, Brugha T, de Graaf R, Bruffaerts R, et al. Risk factors for suicidality in Europe: results from the ESEMED study. J Affect Disord. 2007;101(1-3):27-34.

28. Castillejos MC, Huertas P, Martín P, Moreno KB. Prevalence of suicidality in the European general population: a systematic review and meta-analysis. Arch Suicide Res. 2020. https://doi.org/10.1080/13811118.2020.1765928.

29. Dong M, Wang SB, Li Y, Xu DD, Ungvari GS, Ng CH, Chow IH, Xiang YT. Prevalence of suicidal behaviors in patients with major depressive disorder in China: a comprehensive meta-analysis. J Affect Disord. 2018;225:32-9.

30. Stene-Larsen K, Reneflot A. Contact with primary and mental health care prior to suicide: A systematic review of the literature from 2000 to 2017. Scand J Public Health. 2017:47:1-9.

31. Alem AJL, Kebede D, Kullgren G. Awareness and attitudes of a rural Ethiopian community toward suicidal behaviour A key informant study in Butajira, Ethiopia. Acta Psychiairica Scand Vica. 1999;65(69):1-5.

32. Fekadu AAA, Medhin G, Shibre T, Cleare A, Prince M, Kebede D. Utility of the concept of minor depressive disorder: evidence from a large rural community sample in a developing country setting. J Affect Disord. 2007;104(1-3):111-8.

33. Fekadu AODM, Alem A, Kebede D, Church S, Johns L, Medhin G, Prince $M$, Shibre T. Validity of the concept of minor depression in a developing country setting. J NervMent Dis. 2008;196(1):22-8.

34. Fekadu A, Medhin G, Selamu M, Hailemariam M, Alem A, Giorgis TW, Breuer E, Lund C, Prince M, Hanlon C. Population level mental distress in rural Ethiopia. BMC Psychiatry. 2014;14(194):1-13.

35. Mekonnen DKY. The prevalence of suicidal ideation and attempts among individualsattending an adult psychiatry out-patient clinic in Gondar, Ethiopia. Afr Health Sci. 2011;11(1):103-7.

36. Gelaye B, Lemma S, Deyassa N, Bahretibeb Y, Tesfaye M, Berhane Y, Williams MA. Prevalence and correlates of mental distress among working adults in ethiopia. Clin Pract Epidemiol Ment Health. 2012;8(1):123-33.

37. Gebrekidan Abbay A, Tibebe Mulatu A, Azadi H. Community knowledge, perceived beliefs and associated factors of mental distress: a case study from Northern Ethiopia. Int J Environ Res Public Health. 2018;15(2423):1-22.

38. Alem AKD, Woldesemiat G, Jacobsson L, Kullgren $G$. The prevalence and socio-demographic correlates of mental distress in Butajira, Ethiopia. Acta Psychiatr Scand Suppl. 1999;99(397):48-55.

39. Nyundo A, Manu A, Regan M, Ismail A, Chukwu A, Dessie Y, Njau T, Kaaya SF, Smith Fawzi MC. Factors associated with depressive symptoms and suicidal ideation and behaviours amongst sub-Saharan African adolescents aged 10-19 years: cross-sectional study. Trop Med Int Health. 2019;25:54-69.
40. Bekry AA. Trends in suicide, parasuicide and accidental poisoning in Addis Ababa, Ethiopia. Ethiop J Health Dev. 1999;13(3):247-61.

41. Bifftu BB, Dachew BA, Tiruneh BT, Guracho YD. Prevalence of suicidal ideation, suicidal attempt and completed suicide in Ethiopia: a systematic review and meta-analysis protocol. Syst Rev. 2019;8(72):1-5.

42. Garzotto N, Buglass D, Holding TA, et al. Aspects of suicide and parasuicide. Acta Psychiatr Scand. 1977;56:204-14.

43. Miranda RSM, Hicks R, et al. Suicide attempt characteristics, diagnoses, and future attempts: comparing multiple attempters to single attempters and ideators. J Am Acad Child Adolesc Psychiatry. 2008;47:32-40.

44. Gordon R. An operational classification of disease prevention. In: Steinberg J, Silverman M, editors. Preventing mental disorders: a research perspective. Rockville: US Department of Health and Human Services: National Institute of Mental Health; 1983. p. 20-6.

45. Research loMRRfMDFfPI. In: Mrazek PJ, Haggerty RJ, editors. Committee on Prevention of Mental Disorders, Division of Biobehavorial Sciences and Mental Disorders. Washington, DC: National Academy Press; 1994.

46. Shamseer $L$, Moher D, Clarke M, et al. Preferred reporting items for systematic review and meta-analysis protocols (PRISMA-P) 2015: elaboration and explanation. BMJ. 2015;349:7647.

47. Association AP. DSM-5, diagnostic and statistical manual of mental disorders. 5th ed. Arlington: American Psychiatric Publishing; 2013.

48. Diekstra RFWGW. The epidemiology of suicidal behavior: a review of three continents. World Heath Stat Quar Geneva. 1993;46(1):52-68.

49. Munn Z, Moola S, Lisy K, Riitano D, Tufanaru C. Methodological guidance forsystematic reviews of observational epidemiological studies reportingprevalence and cumulative incidence data. Int J Evid Based Healthc. 2015;13(3):147-53.

50. Berkey CSHD, Mosteller F, Colditz GA. A random-effects regression model for meta-analysis. Stat Med. 1995;14(4):395-411.

51. DerSimonian R, Laird N. Meta-analysis in clinical trials. Control Clin Trials. 1986;7:177-88

52. Higgins JP, Thompson SG. Quantifying heterogeneity in a meta-analysis. Stat Med. 2002;21(11):1539-58.

53. Duval S, Tweedie R. Trim and fill: a simple funnel-plot-based method of testing and adjusting for publication bias in meta-analysis. Biometrics. 2000;56:455-63.

54. Liu JL. The role of the funnel plot in detecting publication and related biases in meta-analysis. Evid Based Dent. 2011;12(4):121-2.

55. Egger MDSG, Schneider M, Minder C. Bias in meta-analysis detected by a simple, graphical test. BMJ (Clinical research ed). 1997;315(7109):629-34.

56. Lee S, Fung S, Tsang A, Liu Z, Huang Y-Q, He Y, Zhang M, Shen Y-C, Nock MK, Kessler RC. Lifetime prevalence of suicide ideation, plan, and attempt in metropolitan China. Acta Psychiatr Scand. 2007;1 16:429-37.

57. Cao XL, Zhong BL, Xiang YT, Ungvari GS, Lai KY, Chiu HF, et al. Prevalence of suicidal ideation and suicide attempts in the general population of China: a meta-analysis. Int J Psychiatry Med. 2015;49(4):296-308.

58. Miranda-Mendizabal A. Gender differences in suicidal behavior in adolescents and young adults: systematic review and meta-analysis of longitudinal studies. Int J Public Health. 2019;64:265-83.

59. Witte TK, Gauthier JM, Huang X, Ribeiro JD, Franklin JC. Is externalizing psychopathology a robust risk factor for suicidal thoughts and behaviors? A meta-analysis of longitudinal studies. J Clin Psychol. 2018;74:1607-25.

60. Belete HME. Suicidal behaviour in postnatal mothers in northwestern Ethiopia: a crosssectional study. BMJ Open. 2019;9:e027449. https://doi. org/10.1136/bmjopen-2018-027449.

\section{Publisher's Note}

Springer Nature remains neutral with regard to jurisdictional claims in published maps and institutional affiliations. 